\section{Expiration Dating Of Chemicals And Reagents}

Rande Kline, EM Science

rkline@emscience.com

With today's requirements for chemicals and reagents in research and clinical laboratory environments, questions often arise concerning expiration dating. Why are not all chemicals and reagents expiration dated by the manufacturer when they are made? How should expiration of chemicals and reagents not dated by the manufacturer be determined? How would a laboratory establish expiration of solutions and reagents prepared in-house?

To assure good manufacturing practices, many manufacturers comply with guidelines set for them by agencies such as the International Organization for Standardization (ISO) and are inspected by ISO auditors. Some manufacturers are required to comply with the Food and Drug Administration (FDA.) These manufacturers are known as GMP facilities (good manufacturing practices) and are assessed by FDA inspectors. Like regulatory agencies who govern clinical sites, ISO and FDA require manufacturers to establish uniform quality systems for product testing and certification.

Manufacturers who are ISO certified have internal process controls which require periodic examination of chemical appearance and specifications at specific times. GMP facilities follow FDA mandated standard operating procedures (SOPs). FDA guidelines recommend expiration dating USP chemicals known to deteriorate in less than two years. All FDA regulated pharmaceuticals and in vitro diagnostics require expiration dating. Raw material chemicals and pure chemicals for lab use often do not require expiration dates.

Shelf life is defined as the period of time a product is considered usable for each application, taking into account physical and $f_{0}^{\circ}$ or chemical changes. Products considered to have extensive shelf life when stored unopened and under the proper conditions are not given an expiration date. To clarify a common misconcep tion, "no expiration date" and "forever" are not synonymous Chemicals are used for a variety of applications. Users should assess a chemical for actual expiration based on the application! for which the chemical or reagent is being employed.

In the laboratory, inspection of appearance and quality on $a^{\infty}$ regular basis or just before use should be a routine procedure $\frac{\tau}{5}$ Most chemicals undergoing deterioration will have distinct physica appearances easily recognizable to users including color change $\stackrel{\circ}{\circ}$ liquefaction, and clumping. Decomposition information is providedF in the Material Safety Data Sheet (MSDS).

Each time a bottle is opened a chemical is exposed to various outside influences which may alter its integrity for a specific use A chemical such as ethylenediamine is an amine which will de compose easily to form crusty, particulate matter and brownish yellow coloration upon exposure to air or heat due to oxidation. This is a natural reaction caused by carbon dioxide from the at mosphere. Ethylenediamine may not be given shelf life by the manufacturer because if unopened and packaged under nitrogen these conditions are eliminated. But, the user should monitor ethylenediamine once the product is opened, even if it is purged with nitrogen or another inert gas after each use.

Humidity from the air affects hygroscopic salts such as Sodium Phosphate Dibasic by adding moisture to the weight thus altering the assay of the product and causing the particles to be-
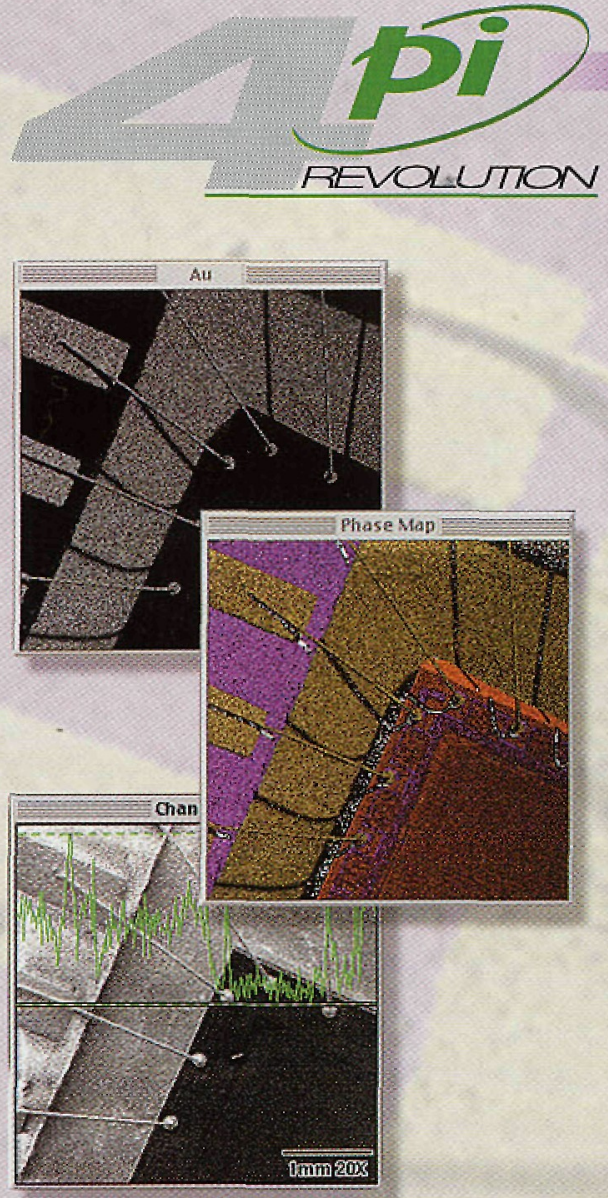

Spectral and Image Accuisition software for Windows ${ }^{\circ}$ and Mac os

- Micron markers

- Easy-to-use point-and-click screen tools

- Preview and acquire images of different sizes

- Multiple spectra windows with independent scaling and colors

- Simultaneous acquisition of SEM, WDS, SCA and X-ray images

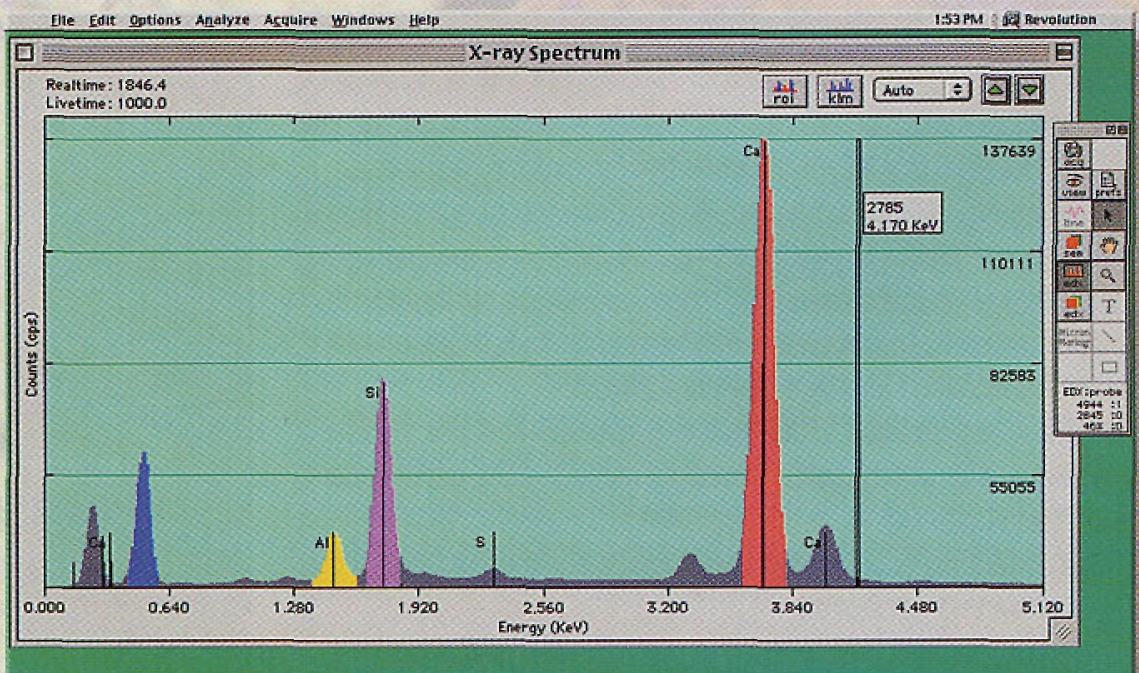

4 pi Analysis, Inc. X-ray detectors, EDS and Imaging Systems and Upgrades (919) 489-1757

sales@4pi.com • www.4pi.com 
come lumpy and hard. If a bottle of Sodium Phosphate Dibasic is opened and used in entirety there would be no concern. If the bottle is opened three times a week over a period of 2 months, the user may need to re-assay the chemical to adjust the current weight by the amount of water absorbed in order to ensure the proper concentration of salt is present for the application being utilized.

Some salts are not affected adversely by humidity. For instance, Sodium Chloride is not as hygroscopic as Sodium Phosphate Dibasic. Frequent exposure to the air does not markedly affect the physical and/or chemical properties, for this reason it could be given expiration of two years or longer by the user and may not require adjustment for concentration.

Laboratories should create their own SOPs for replacement of chemicals and reagents after a specified period of time. Once a chemical is received into a laboratory, a record should be established noting the date received, date opened, and the userdefined date for expiration. Expiration dating should start from the date opened. Chemicals that are purchased with manufacture's expiration dates should follow the same rule, keeping in mind the expiration date is limited to the date listed by the manufacturer.

Some chemicals may terminate for one use but still be suitable for another. For example, Sodium Hydroxide used in a normal solution to adjust $\mathrm{pH}$ to two decimal places may have reached its expiration date for this type of application; however, it would still be suitable to adjust $\mathrm{pH}$ of acids prior to disposal, a less critical application.

A practical beginning step to the process of controlling the quality of chemicals would be to purchase chemicals in quantities fitting the volume for use. Smaller containers are appropriate for low volume use and for products that have short self-life while larger containers may be appropriate for high volume use and products with indefinite shelf-life.

For additional information, contact the manufacturer of the chemical(s) in use to learn more about their view on shelf life and signs of deterioration that may be indicative of a particular chemical.

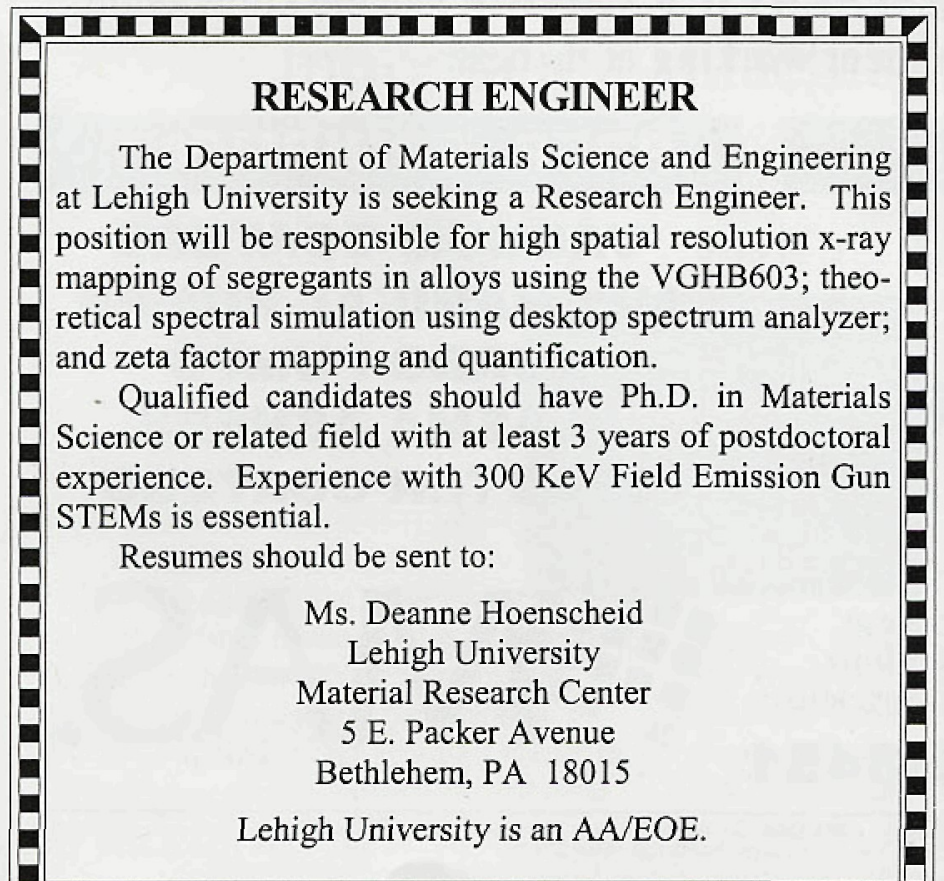

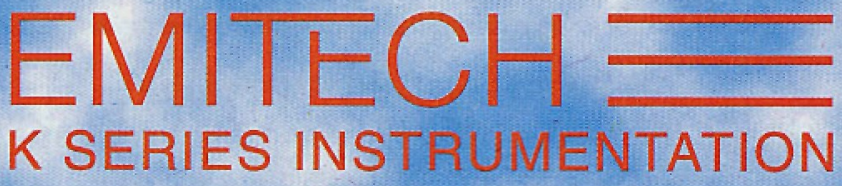

- Extensive range of Consumables $\varepsilon$ Accessories

- Carbon Evaporation Units

Critical Point Driers

Freeze Substitution

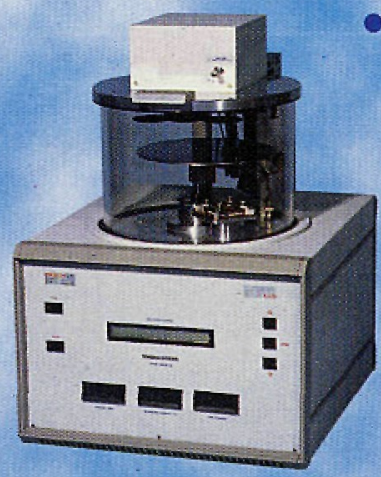

K675 Turbo Pumped Large Sample Chromium Conter, Semi Conductor Applications.

Sputtering Systems

Metal Evaporation

Cryo SEM Systems

- Plasma Asbing

- Freeze Driers
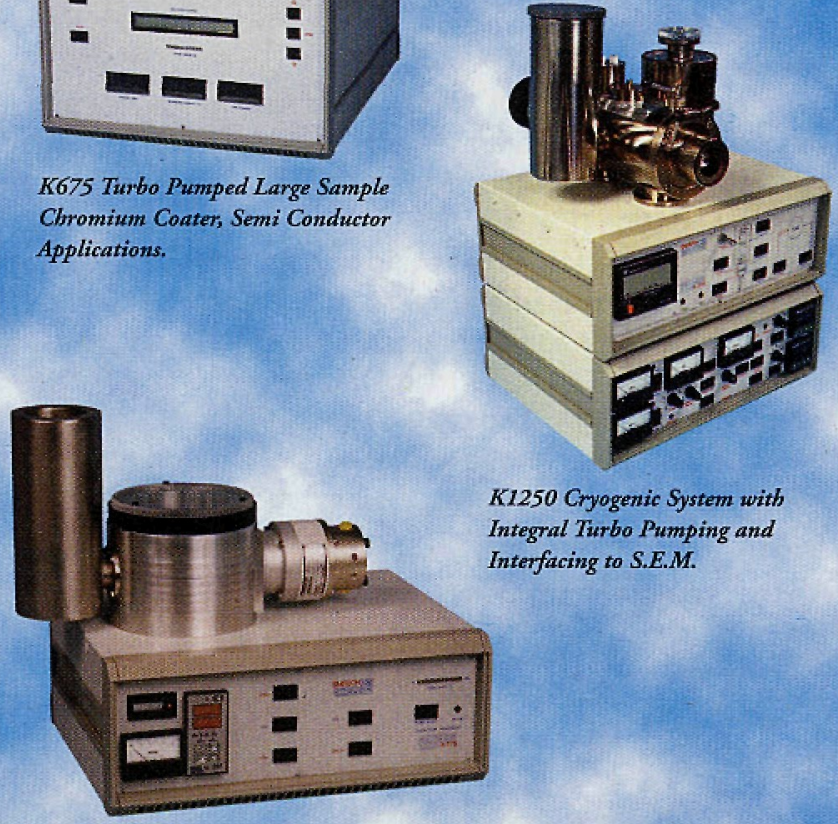

K1250 Cryogenic System with Integral Turbo Pumping and Interfacing to S.E.M.

K775 Turbo Pumped Low Temperature Freeze Drier.

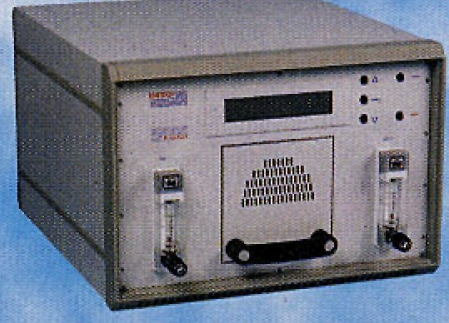

K1050X Plasma Ashing RF System.

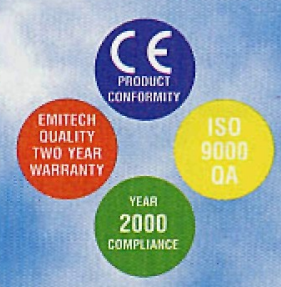
Emitech in U.S.A
Contact: Linda Dailey

888-580-8366

Tel: 2815800568 Fax: 2815800593

\author{
E-mail: emitech@earthlink.net \\ Website: www.emitech.co.uk \\ Emitech Products Inc.
}

